\title{
El valor de la educación en el empleo del sector privado
}

\section{Rafael Gobernado Arribas}

Universidad de Málaga. Departamento de Derecho del Estado y Sociología gobernado@uma.es

\section{Resumen}

Es complicado revalidar empíricamente las diferentes teorías y modelos que explican la relación entre educación y empleo. Para facilitar esta tarea, se propone aquí un conjunto de sucesivas clasificaciones dicotómicas de tales teorías y modelos. La elección entre cada una de las disyuntivas de cada escalón no es difícil. La base de datos en la que se apoya este trabajo es la Encuesta de Calidad de Vida en el Trabajo, de 1999, del Ministerio de Trabajo y Seguridad Social.

Palabras clave: educación, ingresos, mercado de trabajo, puesto de trabajo, organización empresarial.

Abstract. The value of education in the private sector employment

It is complicated to validate (confirm) empirically the different theories and models which explain the relationship between education and employment. To make this task easier, we suggest here a set of dichotomic consecutive classifications of the theories and models mentioned above. It is not difficult to choose between the two difficult options in each stage. This work is based on the 1999 Survey on the Quality of Life at Work, conducted by the Ministry of Labour and Social Security.

Key words: education, income, labour market, job, employers organizations.

\section{Sumario}

1. Objetivos y antecedentes

2. La base de datos y su análisis

3. El modelo liberal versus el modelo nacional-funcional

4. Modelo educativo de producción directa versus producción indirecta

5. Modelo de producción indirecta favorable a la empresa versus el favorable al estatus del empleado
6. Los datos con más detalle

7. La importancia de la educación en el nivel de ingresos. Posibles explicaciones

8. El ajuste con el puesto de trabajo.

9. Epílogo

Bibliografía 


\section{Objetivos y antecedentes}

En el texto que sigue ${ }^{1}$ se estudia la relación entre la educación formal y el empleo. Más concretamente, se da un repaso al interés económico que tiene el nivel educativo en el mercado laboral. La muestra del análisis está formada por la población asalariada española del sector privado en el último cambio de siglo.

Las teorías y los modelos teóricos que tratan de explicar la relación entre educación y empleo son muchos y muy variados. Existen buenos resúmenes de tales explicaciones (véase, por ejemplo: M. I. García Espejo, 1998: 1-62; I. Brunet y A. Morell, 1998; R. Rubinson e I. Browne, 1994; S. M. Ruesga Benito, J. M. García de la Cruz y C. Muruyama Rendón, 2000), por lo que no merece la pena extenderse sobre ello. Se debe recordar, no obstante, que revalidar empíricamente la mayor parte de esos modelos es sumamente complicado. Para facilitar esa tarea de revalidación empírica en el presente estudio, partimos de sucesivas clasificaciones dicotómicas de tales modelos y teorías según sus enfoques teóricos más generales. De esta manera, se limita el esfuerzo a la elección de un modelo entre pares complementarios, en cada escalón clasificatorio.

Los pares teóricos complementarios son los siguientes: en primer lugar, se pueden clasificar esas teorías y modelos en dos grandes categorías o enfoques teóricos de acuerdo con P. Tripier (1995: 151-168): la interpretación liberal y la interpretación nacional-funcional. Más o menos viene a decir este autor que caben dos criterios para ubicar a los empleados en cada puesto de trabajo: el de la empresa (interpretación liberal) y el del sistema educativo del Estado correspondiente. En otras palabras, la ubicación es un proceso guiado por directrices internas de cada empresa o, por el contrario, el proceso se guía por criterios externos y generales para toda la población del Estado. En el enfoque teórico liberal, la ubicación laboral depende de criterios internos de la empresa. Son las necesidades productivas y organizacionales las que deciden en general la ubicación de cada empleado. Por el contrario, el enfoque nacional-funcional depende del sistema educativo del país correspondiente, y la clasificación laboral consecuente es resultado del mismo. El desenlace es un sistema ocupacional y remunerativo homogéneo para todo el Estado. La realidad seguramente constituye una mezcla de ambas influencias: la del sistema educativo sobre la organización empresarial y la de ésta sobre aquél ${ }^{2}$. Nuestro objetivo, en este caso, es constatar qué enfoque teórico de los citados tiene más importancia en el mercado laboral español.

A su vez, en segundo lugar, es posible clasificar el enfoque nacional-funcional (el que se basa en la educación) en otros dos tipos. En otras palabras,

1. Este trabajo se ha realizado gracias a la colaboración del Plan Propio de Investigación de la Universidad de Málaga.

2. D. K. Müller (1992) estudió el impacto que tienen las calificaciones académicas en la jerarquía socioprofesional de la Alemania del siglo XIX. Se trata de un caso extremo de convergencia entre el sistema educativo y el sistema productivo. El sistema educativo terminó por conformar las posiciones internas no sólo en la Administración pública, sino también en la empresa privada alemana. 
se puede interpretar el valor de la educación para la empresa de dos formas diferentes (M. I. García Espejo, 1998: 8-41). Una de ellas supone que la educación proporciona habilidades y conocimientos directamente relacionados con la productividad. Según esto, los empleados con más años de escolaridad son más productivos que los que tengan menos años. Las teorías del capital humano (G. Becker, 1983) y el funcionalismo tecnológico (B. Clark, 1962) participan de esta creencia.

La otra forma de interpretar la educación consiste en negar tal relación directa entre escolaridad y productividad. La escolaridad es un indicador indirecto de otras cosas: de estatus, de capacidad intelectual, de habilidades comunicativas, de capacidad de aprendizaje, de autodisciplina, de sometimiento a una jerarquía, etc. El aprendizaje de lo que necesita el puesto de trabajo se realiza en el mismo puesto de trabajo. La teoría del filtro educativo (K. Arrow, 1991), la teoría de la correspondencia (S. Bowles y H. Gintis, 1985), la teoría de colas (L. C. Thurow, 1977) y la teoría credencialista (R. Collins, 1989) se inclinan más por esta otra forma de interpretar la escolaridad. Al igual que con la clasificación anterior y con la siguiente, pretendemos apreciar cuál de estos otros dos enfoques tiene más que ver con la realidad laboral española.

En tercer lugar, dentro de este enfoque general que niega la relación directa de la educación con la productividad en el puesto de trabajo, caben otras dos posibilidades. En primer lugar, aquélla que entiende que la educación es una señal de capacidad de aprendizaje, de capacidad de producción en el futuro o de cualquier otro tipo de utilidad para la empresa (como, por ejemplo, facilitar la selección del personal). Frente a esta concepción se alza aquélla otra que ve a la educación como un simple mecanismo de transmisión de estatus familiar que permite el acceso a un puesto de trabajo que facilita, de paso, la permanencia en tal estatus. La utilidad no es para la empresa, sino para el empleado y su familia. Ésta es la idea general que defiende R. Collins y su teoría credencialista.

Por su parte, el enfoque liberal (el del empresario que se guía por consideraciones internas de la empresa) admite dos tipos de mercado laboral en función de la libertad del propio empresario a la hora de emplear y controlar a los trabajadores. Aunque la terminología varía, la idea que subyace es semejante: hay una proporción de puestos de trabajo que son ventajosos para los empleados y otra proporción que no son ventajosos. En la primera proporción el empresario carece de poder de decisión y en la segunda sí tiene poder. Se habla, así, de conceptos complementarios tales como: sector primario y sector secundario del mercado de trabajo (M. J. Piore, 1999), mercados internos de trabajo y mercados normales de trabajo (P. B. Doeringer y M. J. Pioré, 1999; R. C. Edwards, 1999), mercados inflexibles y mercados flexibles. Esta última clasificación escapa a mi interés en el presente análisis.

Por supuesto, como ya se dijo, la realidad es una mezcla compleja de todas estas posibilidades. Cada enfoque debe entenderse como resultado de abstraer de la realidad aquellos aspectos que el modelo considera. Se trata, por lo tanto, 
de abstracciones que poseen su parte de "verdad». El objetivo inmediato del texto que sigue es determinar si la ubicación en los puestos de trabajo en España en la actualidad se explica más por uno que por otro de los criterios indicados arriba.

Se pretende, por lo tanto, buscar las proporciones en las que tales explicaciones se mezclan. Esas proporciones no son estables a lo largo del tiempo, y en cada país varían respecto a las demás. Por ejemplo, en el primer caso, el que distingue entre el modelo liberal y el nacional-funcional, influye la situación propia del ciclo económico (P. Tripier, 1995: 157 y 158): en épocas de crecimiento económico acusado toma importancia el criterio nacional-funcional; mientras que en épocas de recesión gana peso el criterio liberal. Es decir que el universalismo, más abundante en el primer criterio, es sustituido por el particularismo, más propio del criterio liberal. Así mismo, la expansión educativa influye necesariamente en contra del criterio nacional-funcional (R. Boudon, 1977), puesto que el nivel educativo deja de ser discriminante a la hora de buscar empleo. Es de presumir, además, que dentro de un mismo Estado y una misma época se aprecien diferencias importantes entre las distintas categorías ocupacionales ${ }^{3}$.

3. Pese a la redacción de algunas frases, el objetivo nuestro no es medir la productividad en función de las categorías educativas u organizacionales. Nuestro objetivo es conocer los mecanismos de distribución de la renta, los mecanismos que generan desigualdad salarial en el mercado de trabajo. En general, los sociólogos están más interesados por el análisis de la desigualdad social que por el de la producción.

La productividad como explicación del monto de salario se acepta como hipótesis posible en algunas de las categorías teóricas aquí usadas, no en todas. Pero es una hipótesis cuya falsación, llegado el caso, no trasciende al resultado del trabajo.

No obstante, la economía neoclásica entiende que si una empresa pretende maximizar sus beneficios, los trabajadores deben recibir un salario equivalente a su producto marginal. En principio, por lo tanto, existe una obvia relación entre la productividad y los ingresos. La duda es si las empresas desean maximizar o no sus beneficios. Por su parte, la función de productividad depende del capital físico, de la fuerza del trabajo y del capital humano (véase M. L. Katz y H. L. Rosen, 1997: 256-259). De ahí incluso que algunos trabajos sobre capital humano utilicen el nivel educativo como indicador indirecto de productividad. De cualquier forma, es habitual el uso de los salarios como indicadores de productividad (véase, entre otros, I. Corugedo, 1995; A. H. Goldsmith y otros, 2000; L. Serrano, 1996).

Por su parte, la tradición sociológica ha adoptado dos posiciones contrapuestas sobre la hipótesis que identifica salario con productividad. De acuerdo con la teoría funcionalista de Davis y Moore, de finales de la década de 1940, los ingresos dependen de la utilidad (productividad en el sentido más amplio del término) que redunde el empleado a la empresa. Cuanta más utilidad, mayores ingresos. Las críticas a tal planteamiento no se hicieron esperar. Las razones del tamaño de los ingresos son múltiples y entre ellas destacan las relaciones de poder. Melvin Tumin, funcionalista también, se encargó de argumentar la crítica a los anteriores autores. El debate de la época se describe con brillantez en J. Díez Nicolás y J. del Pino Artacho (1972). Parece ser que la tesis de Davis y Moore se cumple aceptablemente en los tramos intermedios de distribución de la renta, que es nuestro caso, mientras que no se cumple entre la población que ocupa los extremos de esa distribución. 


\section{La base de datos y su análisis}

La fuente de datos que se ha utilizado es la Encuesta de Calidad de Vida en el Trabajo, de 1999, realizada por el Ministerio de Trabajo y Seguridad Social en junio de ese mismo año. La encuesta se realizó sobre una muestra de seis mil personas ocupadas. La muestra ha sido convenientemente ponderada por los propios autores de la encuesta según sexo, edad, comunidad autónoma y tamaño del municipio, siguiendo los datos de la Encuesta de Población Activa del primer trimestre de 1999. Representa, por lo tanto, con bastante precisión a la población ocupada española del citado año.

$\mathrm{El}$ «nivel educativo del entrevistado» es una de las variables más considerada en el análisis. A partir de la presentación de la misma en el cuestionario, hemos elaborado otras dos variables: «años de escolaridad del entrevistado» y "nivel educativo del entrevistado». Para conseguir la variable «años de escolaridad» se ha traducido cada nivel educativo a la cantidad de años oficiales que se tarda en obtener la titulación correspondiente. Se han aplicado los criterios utilizados ya por la Encuesta Sociodemográfica de 1991 (J. Caravaña, 1999: 282-283). Los resultados oscilan entre 20 años (título de doctor y estudios de posgrado universitarios) y 0 (los analfabetos).

Por su parte, los diferentes niveles educativos propuestos por la encuesta han sido resumidos en tres: estudios primarios, secundarios y superiores. En este caso se ha prescindido de las categorías «sin estudios» y "analfabetos».

El cuestionario, además, indaga, en su pregunta octava, sobre el «tipo de puesto en la organización» y proporciona tres posibles respuestas: «dirección», «supervisión» y «empleado». Por su parte, la antigüedad en la empresa se ha clasificado en tres categorías: «reciente» (contratados después de 1990), «media» (contratados entre 1975 y 1989) y «antigua» (contratados antes de 1975).

Las variables citadas arriba (niveles educativos, tipo de puesto en la organización y antigüedad en la empresa) constituyen las variables independientes clave, utilizadas a continuación en los análisis de medias de ingresos. Estas tres variables y los respectivos análisis de medias de ingresos van a permitir constatar la importancia relativa de cada una de las disyuntivas presentadas.

Para facilitar la lectura de esos análisis, nos hemos limitado, en general, a utilizar el coeficiente de determinación de los ingresos (c. d.) según: a) los tres niveles de estudio (primario, medio y superior); b) los tres tipos de puesto en la organización (dirección, supervisión y empleado); y c) los tres grados de antigüedad en la empresa (reciente, media, antigua). Se utiliza el coeficiente de variación (c. v.) como medida de dispersión.

El nivel de ingresos mensuales del entrevistado, así como el tamaño del hábitat y el de la empresa en la que trabaja, se han calculado a partir de la marca de clase de las categorías propuestas en el cuestionario. Además, y al igual que con el nivel educativo, estas variables se han recodificado en otras tantas de carácter cualitativo. Hay tres tipos de ingresos. «altos» (más de 350.000 pts. 
al mes), «medios» (entre 150.001 y 350.000) y «bajos» (menos de 150.001). De la misma forma, hay tres tipos de hábitat: «grande» (con más de 250.000 habitantes), «medio» (entre 10.001 y 250.000) y «pequeño» (menos de 10.001). Por su parte, hay tres tipos de empresa: «grande» (con más de 99 empleados), «mediana» (entre 10 y 99) y "pequeña» (menos de 10 empleados). Se han establecido tres grupos de edad: los «jóvenes» (menores de 30 años), los «adultos» (entre 31 y 50 años) y los «mayores» (más de 50 años).

Como se dijo arriba, hemos limitado el estudio a la población asalariada en el sector privado. Se prescinde, salvo cuando se advierta, de los asalariados del sector público y de los empresarios, profesionales y autónomos, así como de la ayuda familiar. Tampoco forman parte del análisis la población desocupada ni la población no activa. Por razones de tamaño muestral, sólo se informará de las diferencias de edad y sexo en el epígrafe de resultados generales.

\section{El modelo liberal versus el modelo nacional-funcional}

La observación empírica de la importancia del criterio liberal y del nacional-funcional no es complicada (P. Tripier, 1995: 163). De acuerdo con el punto de vista liberal (según el cual el empresario es el que decide el puesto de trabajo del empleado), el salario ${ }^{4}$ dependerá sobre todo del empleo o de la categoría ocupacional que ocupe el empleado. Por el contrario, para el enfoque nacionalfuncional (según el cual el puesto de trabajo del empleado depende de factores externos a la empresa), el salario dependerá sobre todo del nivel de estudios del empleado.

Los datos de las tablas 1 y 2 informan que el puesto en la empresa (dirección, supervisión o empleado) explica mucho mejor las desigualdades de salario que los niveles educativos. El coeficiente de determinación es un buen indicador de lo señalado. Los tres tipos de puesto en la empresa «explican» el $17 \%$ de las diferencias de ingresos; mientras que los tres niveles educativos sólo lo hacen en un $8 \%$. Esto permite afirmar que las desigualdades salariales se deciden principalmente según criterios internos de la empresa.

4. Las explicaciones de las diferencias salariales son múltiples y de carácter complejo. Entre otras causas, destacan: la formación y la experiencia, el origen social, la costumbre, las normas colectivas, la estructura de los mercados, los sindicatos y demás organizaciones, etc. (véase D. Marsden, 1994 y H. Ph. Brown, 1990). No obstante, el nivel educativo y la organización interna de la empresa, dentro de un marco de oferta y demanda de empleo, explican buena parte de las diferencias salariales. 
Tabla 1. Medias de ingresos mensuales (pesetas) según niveles educativos. Asalariados del sector privado

\begin{tabular}{lccc}
\hline $\begin{array}{l}\text { Nivel } \\
\text { educativo }\end{array}$ & $\begin{array}{c}\text { Media de } \\
\text { ingresos }\end{array}$ & N & C. V. \\
\hline Primaria & 128.098 & 1.582 & $46 \%$ \\
\hline Secundaria & 137.941 & 653 & $49 \%$ \\
\hline Superior & 201.833 & 441 & $70 \%$ \\
\hline Total & 142.660 & 2.676 & $59 \%$ \\
\hline
\end{tabular}

Coeficiente de determinación: 8\% $(\mathrm{F}=147,5$; significación $=0,000)$.

Fuente: Encuesta de Calidad de Vida en el Trabajo, de 1999.

Elaboración propia.

Tabla 2. Medias de ingresos mensuales (pesetas) según el puesto en la empresa. Asalariados del sector privado

\begin{tabular}{lcccc}
\hline $\begin{array}{l}\text { Puesto en } \\
\text { la empresa }\end{array}$ & $\begin{array}{c}\text { Media de } \\
\text { ingresos }\end{array}$ & N & C. V & $\begin{array}{c}\text { c. d. 1 } \\
\text { (educación) }\end{array}$ \\
\hline Dirección & 300.627 & 74 & $56 \%$ & $19 \%^{*}$ \\
\hline Supervisión & 198.956 & 311 & $54 \%$ & $11 \%^{*}$ \\
\hline Empleado & 128.846 & 2.384 & $51 \%$ & $3 \%^{*}$ \\
\hline Total & 141.328 & 2.769 & $60 \%$ & $8 \%^{*}$ \\
\hline
\end{tabular}

*Significación de la $F<0,001$.

Coeficiente de determinación: $17 \%(F=283,7$; significación $=0,000)$.

Fuente: Encuesta de Calidad de Vida en el Trabajo, de 1999.

Elaboración propia.

La última columna de la tabla 2 proporciona información interesante: el coeficiente de determinación de la educación sobre los ingresos laborales, en cada una de las tres categorías organizacionales. Según esos datos, la educación es mucho más rentable para los que ocupan cargos directivos (coeficiente de determinación $=19 \%)$ que para los empleados $(3 \%)$. Esto significa que el modelo liberal se cumple sobre todo en los niveles organizacionales inferiores, los que tienen menos responsabilidad en la empresa. Por el contrario, en los niveles organizacionales superiores, se impone el modelo nacional-funcional.

La tabla 3 describe la interrelación entre los factores internos de la empresa y los externos ${ }^{5}$. No se aprecia una correlación acusada entre ellos. Se nota

5. Esto nos conduce al tema de las desigualdades sociales. O. Wright (1994) desarrolló un modelo explicativo de la desigualdad al que denominó «modelo de explotaciones múltiples» y que se basa en la conjunción de los dos enfoques citados. Este autor clasifica las ocupaciones 
Tabla 3. Tipos de puesto en la empresa según niveles de educación.

Asalariados del sector privado

\begin{tabular}{lcccc}
\hline $\begin{array}{l}\text { Puesto en } \\
\text { la empresa }\end{array}$ & $\begin{array}{c}\text { Educación } \\
\text { superior }\end{array}$ & $\begin{array}{c}\text { Educación } \\
\text { secundaria }\end{array}$ & $\begin{array}{c}\text { Educación } \\
\text { primaria }\end{array}$ & Total \\
\hline Dirección & 2,1 & 0,7 & 0,9 & $3,7(114)$ \\
\hline Supervisión & 3,4 & 3,4 & 5,4 & $12,1(372)$ \\
\hline Empleado & 11,9 & 20 & 52,2 & $84,2(2.583)$ \\
\hline Total & 17,4 & 24,1 & 58,5 & $100(3.069)$ \\
\hline
\end{tabular}

Coeficiente de contingencia: 0,24 (significación: 0,000).

Fuente: Encuesta de Calidad de Vida en el Trabajo, de 1999.

Elaboración propia.

sobre todo comparando los totales de la tabla, por ejemplo: la proporción de cargos superiores $(3,7 \%)$ es inferior, con mucho, a la proporción de asalariados con estudios universitarios $(17,4)$. De cualquier forma, no tiene por qué coincidir el nivel educativo con el puesto en la jerarquía organizacional. No obstante, se observa un cierto grado de sobreeducación ${ }^{6}$, al menos en la categoría de empleados.

de los asalariados siguiendo dos criterios. En primer lugar, clasifica las ocupaciones según la capacidad de gestión o, lo que es igual, por la cantidad de subordinados que se tiene a cargo. Este es un criterio totalmente interno de la empresa. El otro criterio está formado por los niveles de calificación del empleado. La calificación se define sobre todo por el nivel educativo. Por lo tanto, es un criterio ajeno a la empresa. Se puede entender el modelo de Wright como otro intento de conjugar los dos enfoques aludidos. El interés para nosotros estriba en que la observación de los datos se guía por este modelo, aunque con diferente interpretación.

J. J. González (1992) llevó a cabo un análisis con datos españoles de 1990 basándose en la clasificación de O. Wright. De tal análisis sacamos estos datos sobre medias relativas de ingresos según las categorías utilizadas arriba. Los ingresos más altos son los de puestos directivos con nivel educativo superior (valor $=100$ ).

\begin{tabular}{lccc}
\hline $\begin{array}{l}\text { Puesto en } \\
\text { la empresa }\end{array}$ & $\begin{array}{c}\text { Estudios } \\
\text { superiores }\end{array}$ & $\begin{array}{c}\text { Estudios } \\
\text { medios }\end{array}$ & $\begin{array}{c}\text { Estudios } \\
\text { primarios }\end{array}$ \\
\hline Dirección & 100 & 75 & 58 \\
\hline Supervisión & 61 & 52 & 41 \\
\hline Empleado & 46 & 47 & 33 \\
\hline
\end{tabular}

Fuente: J. J. González (1992). $N=3.390$.

6. El fenómeno de la sobreeducación ha sido abundantemente señalado en España. Ll. Fina Sanglas y otros (2000: 104, 146 y 149) informan de la polarización de la pirámide educativa española, con un exceso de titulaciones superiores e inferiores, y con un déficit de las intermedias, que son las que más necesita el sistema productivo. Por su parte, L. Garrido (1991) llama la atención sobre el hecho de que el sistema educativo genera más titulados superiores de los que necesita el sistema productivo. 


\section{Modelo educativo de producción directa versus producción indirecta}

El siguiente paso consiste en distinguir la parte debida a la productividad que genera directamente la educación formal de la parte generada de forma indirecta por tal educación formal. La fórmula más sencilla es comparar la proporción de ingresos debida al nivel educativo con la proporción de ingresos debida a la antigüedad en la empresa (P. Tripier, 1995: 160). Se supone que si alguien es contratado por criterios educativos indirectos, producirá más cuanto más tiempo esté en la empresa y aprenda sus tareas en el puesto de trabajo. Además, es presumible también que la antigüedad en la empresa es mucho más aprovechable para los que tienen nivel educativo alto. Esto es así porque el nivel educativo es, en este caso, una señal de la facilidad de aprendizaje en el puesto de trabajo. Por el contrario, si la productividad se debe directamente a la educación, los ingresos no tienen por qué variar mucho a lo largo de la carrera profesional, ya que el nivel educativo tampoco cambia.

De acuerdo con los datos del total de la población de la tabla 4, la antigüedad en la empresa tiene tanto interés como el nivel educativo a la hora de fijar los salarios. El coeficiente de determinación correspondiente es el 8\%, al igual que en el caso de la educación. Una primera lectura induce a sospechar que la elección del personal se sigue de acuerdo con los dos criterios: la educación formal es directamente productiva, al mismo tiempo que se elige pensando en la capacidad de aprendizaje o utilidad posterior del nivel educativo del asalariado. De cualquier forma, ambos criterios juntos tienen menor capacidad explicativa de los salarios que la organización interna de la empresa.

Tabla 4. Medias de ingresos mensuales (pesetas) según antigüedad en la empresa. Asalariados del sector privado

\begin{tabular}{lcccc}
\hline $\begin{array}{l}\text { Antigüedad en } \\
\text { la empresa }\end{array}$ & $\begin{array}{c}\text { Media de } \\
\text { ingresos }\end{array}$ & $\mathrm{N}$ & C. V. & $\begin{array}{c}\text { c. d. 1 } \\
\text { (educación) }\end{array}$ \\
\hline Reciente & 125.233 & 1.856 & $52 \%$ & $8 \%^{*}$ \\
\hline Media & 172.174 & 607 & $58 \%$ & $18 \%^{*}$ \\
\hline Antigua & 188.686 & 242 & $58 \%$ & $23 \%^{*}$ \\
\hline Total & 141.448 & 2.704 & $58 \%$ & $8 \%^{*}$ \\
\hline
\end{tabular}

Significación de la $F<0,001$.

Coeficiente de determinación: 8\% $(F=130,8$; significación $=0,000)$.

Fuente: Encuesta de Calidad de Vida en el Trabajo, de 1999.

Sin embargo, los datos de la última columna de la tabla 4 indican una clara preponderancia del modelo de producción indirecta sobre el de producción directa. Dice aquel modelo que el nivel educativo no es otra cosa que un indicador de aprendizaje o de adaptación al puesto de trabajo. Ese aprendizaje o adaptación implica un proceso de mejora más acusado para los que tienen niveles edu- 
cativos altos que para los demás. Los coeficientes de determinación indican que los empleados más antiguos rentabilizan más sus estudios que los recientes, lo que encaja plenamente con esa idea ${ }^{7}$ : el $23 \%$ de la varianza de ingresos de los empleados más antiguos tiene que ver con el nivel educativo; mientras que sólo tiene que ver con un $8 \%$ entre los más recientes.

\section{Modelo de producción indirecta favorable a la empresa versus el favorable al estatus del empleado}

El modelo credencialista interpreta la relación entre educación y empleo de forma indirecta. Según este modelo, la productividad no está ligada a la educación. La educación está ligada al estatus. El estatus proporciona una cultura específica que facilita el acceso a los empleos mejor remunerados. La educación sirve para identificar el estatus. Ahora bien, la cultura de estatus se adquiere en la familia, es una cuestión de "herencia cultural» entre padres e hijos. Por ello, un indicador de vigencia del modelo credencialista es la posible relación entre las dos variables siguientes: a) el coeficiente de determinación del nivel educativo sobre los ingresos y b) la movilidad social intergeneracional.

Los datos que tenemos no revalidan la idea credencialista. La movilidad de clase, o la falta de ella, no es discriminante en absoluto en cuanto al interés del nivel educativo sobre los ingresos. No hay apenas diferencia en el coeficiente de determinación del nivel educativo sobre los ingresos entre los que heredan del padre la clase ocupacional y los que cambian de clase. Los que heredan la clase ocupacional obtienen un coeficiente de determinación del $9 \%$, frente a un $8 \%$ de los que han cambiado de clase con respecto al padre.

Además, de acuerdo con los datos de la tabla 8, la correlación entre el coeficiente de determinación citado (columna primera de la tabla 8) y la herencia intergeneracional de clase ocupacional (fila 4), de cada una de las categorías ocupacionales estudiadas, es muy baja e incluso negativa: $-0,32$. Se puede afirmar, por lo tanto, que en la actualidad la importancia económica de la educación no depende de la herencia de estatus ${ }^{8}$.

La herencia de clase ocupacional tampoco parece que tenga mucho que ver con el coeficiente de determinación de los ingresos según el tipo de puesto (dirección, supervisión o empleado) en la empresa.

7. El hecho de que los más antiguos en la empresa son los que más aprovechan el rendimiento económico de la educación, puede explicarse de otras formas. Seguramente también tiene que ver esta situación con la escasez de títulos académicos en épocas pretéritas, lo que les hacía ser más rentables. También puede ser entendida la importancia del título como una defensa de los más antiguos frente a los nuevos (P. Tripier, 1995: 160).

8. Estos datos se repiten en los análisis de logro ocupacional: la influencia (directa e indirecta) del origen social sobre los ingresos del entrevistado es inferior a la influencia del nivel educativo y de la ocupación del propio entrevistado. Véase J. Caravaña (1999: 333). 


\section{Los datos con más detalle}

La tabla 5 compara los resultados de los asalariados del sector privado con otras situaciones profesionales. Lo más significativo de la tabla es la diferencia entre los asalariados del sector público y los del sector privado. En éstos, como ya se dijo, influye más en los ingresos el tipo de puesto en la empresa que el nivel educativo; mientras que en los asalariados del sector público lo que influye más en los ingresos es el nivel educativo, y mucho menos el tipo de puesto que se ocupe en la organización. La antigüedad en la empresa en los funcionarios apenas tiene trascendencia económica. Esto induce a pensar que en el sector público, el criterio preponderante es el nacional-funcional, así como que los conocimientos adquiridos en la educación formal son directamente aplicables en el puesto de trabajo.

Los empresarios con asalariados constituyen la categoría ocupacional que tiene ingresos más altos y también es la categoría en la que el nivel de estudios influye más en los mismos (c. d. $1=17 \%$ ). Por el contrario, y como ya se sabía, los ingresos de los trabajadores por cuenta propia sin asalariados son casi independientes del nivel de estudios.

Tabla 5. Coeficientes de determinación del nivel educativo (c. d. 1) y del tipo de puesto de trabajo (c. d. 2) sobre los ingresos, según la situación profesional.

Asalariados del sector privado

\begin{tabular}{|c|c|c|c|c|c|c|c|c|c|}
\hline $\begin{array}{l}\text { Situación } \\
\text { profesional }\end{array}$ & $\begin{array}{l}\text { Media } \\
\text { ingresos }\end{array}$ & c. v. & $\mathrm{N}$ & $\begin{array}{c}\text { Media } \\
\text { años } \\
\text { escolaridad }\end{array}$ & c. v. & $\mathrm{N}$ & $\begin{array}{c}\text { c. d. } 1 \\
\text { (educac.) }\end{array}$ & $\begin{array}{c}\text { c. d. } 2 \\
\text { (organiz.) }\end{array}$ & $\begin{array}{l}\text { c. d. } 3 \\
\text { (antig.) }\end{array}$ \\
\hline $\begin{array}{l}\text { Asalariados } \\
\text { sector público }\end{array}$ & 186.258 & $58 \%$ & 839 & 12,3 & $37 \%$ & 987 & $10 \% *$ & $5 \% *$ & $3 \% *$ \\
\hline $\begin{array}{l}\text { Asalariados } \\
\text { sector privado }\end{array}$ & 142.660 & $59 \%$ & 2.676 & 9,3 & $45 \%$ & 3.331 & $8 \% *$ & $17 \% *$ & $8 \% *$ \\
\hline $\begin{array}{l}\text { Empresarios } \\
\text { con asalariados }\end{array}$ & 236.943 & $79 \%$ & 157 & 10 & $47 \%$ & 224 & $17 \% *$ & - & - \\
\hline $\begin{array}{l}\text { Profesionales } \\
\text { y autónomos } \\
\text { sin asalariados }\end{array}$ & 180.585 & $73 \%$ & 426 & 8,4 & $52 \%$ & 426 & $2 \% *$ & - & - \\
\hline
\end{tabular}

*Significación de la $F<0,001$.

Fuente: Encuesta de Calidad de Vida en el Trabajo, de 1999.

Elaboración propia.

En el sector privado, el salario de las mujeres ocupadas por cuenta ajena tiene poco que ver tanto con la educación como con la organización de la empresa. Los respectivos coeficientes de determinación son menores que los de los varones. La antigüedad en la empresa condiciona el salario en igual medida en ambos sexos. Existen otros condicionamientos no contemplados 
aquí y que tienen menos fuerza entre los varones 9 . Dentro de esos límites, los ingresos de las mujeres dependen más del nivel de estudios, mientras que los de los varones dependen más del puesto que se ocupe en la organización (tabla 6).

En todas las categorías de edad de los asalariados del sector privado, la organización empresarial tiene más peso en los ingresos que el nivel de estudios. Así mismo, cuanta más edad se tenga, mayor es la dependencia de los ingresos respecto al tipo de puesto que se ocupe en la empresa. El salario de los jóvenes, por su parte, apenas parece depender del nivel de estudios (c. d. $=2 \%)$ y poco más de la organización empresarial (c. d. = 7\%) (tabla 6).

Tabla 6. Coeficientes de determinación del nivel educativo (c. d. 1) y del tipo de puesto de trabajo (c. d. 2) sobre los ingresos, según categorías demográficas.

Asalariados del sector privado

\begin{tabular}{lcccccccc}
\hline $\begin{array}{l}\text { Categoría } \\
\text { social }\end{array}$ & $\begin{array}{c}\text { Media } \\
\text { ingresos }\end{array}$ & c. v. & $\mathrm{N}$ & $\begin{array}{c}\text { Media } \\
\text { años } \\
\text { escolaridad }\end{array}$ & c. v. & $\begin{array}{c}\text { c. d. 1 } \\
\text { (educac.) }\end{array}$ & $\begin{array}{c}\text { c. d. 2 } \\
\text { (organiz.) }\end{array}$ & $\begin{array}{c}\text { c. d. 3 } \\
\text { (antig.) }\end{array}$ \\
\hline Varones & 171.321 & $65 \%$ & 1.315 & 9 & $46 \%$ & $11 \%^{*}$ & $18 \% *$ & $7 \% *$ \\
\hline Mujeres & 110.791 & $56 \%$ & 1.143 & 10 & $42 \%$ & $8 \%^{*}$ & $7 \% *$ & $7 \% *$ \\
\hline Jóvenes & 120.804 & $58 \%$ & 1.231 & 10,3 & $36 \%$ & $2 \%^{*}$ & $7 \% *$ & $0 \% *$ \\
\hline Adultos & 164.167 & $64 \%$ & 1.761 & 9,3 & $45 \%$ & $13 \%^{*}$ & $16 \% *$ & $7 \% *$ \\
\hline Mayores & 183.390 & $73 \%$ & 466 & 7,3 & $59 \%$ & $13 \%^{*}$ & $20 \% *$ & $4 \% *$ \\
\hline
\end{tabular}

* Significación de la $F<0,001$.

Fuente: Encuesta de Calidad de Vida en el Trabajo, de 1999.

Elaboración propia.

Por su parte, en la tabla 7 se informa sobre otras categorías ocupacionales. En casi todas estas categorías, el puesto en la organización explica en mayor proporción los ingresos que el nivel educativo del empleado. No obstante, se debe particularizar algo más la información:

- El nivel educativo no tiene ningún interés económico en los contratados discontinuos y casi ninguno en los eventuales.

- Algo parecido ocurre en la rama de actividad del sector primario: agricultura y ganadería.

- En relación con lo anterior, el hábitat pequeño tampoco favorece la rentabilidad de los estudios.

9. A. Peinado López (1988) calcula el efecto discriminación del salario de las mujeres respecto al de los hombres en un $14 \%$ menos. Aunque la diferencia total es del $21 \%$, el $7 \%$ restante se explica por otras variables tales como la menor edad y menor antigüedad en la empresa de las mujeres. 
Tabla 7. Coeficientes de determinación del nivel educativo (c. d. 1) y del tipo de puesto de trabajo (c. d. 2) sobre los ingresos, según categorías ocupacionales.

Asalariados del sector privado

\begin{tabular}{lcccccccc}
$\begin{array}{l}\text { Categoría } \\
\text { ocupacional }\end{array}$ & $\begin{array}{c}\text { Media } \\
\text { ingresos }\end{array}$ & c. v. & $\mathrm{N}$ & $\begin{array}{c}\text { Media } \\
\text { años } \\
\text { escolaridad }\end{array}$ & $\begin{array}{c}\text { c. v. } \\
\text { c. d. 1 } \\
\text { (educac.) }\end{array} \begin{array}{c}\text { c. d. 2 } \\
\text { (organiz.) }\end{array}$ & $\begin{array}{c}\text { c. d. 3 } \\
\text { (antig.) }\end{array}$ \\
\hline RAMA DE ACTIVIDAD & & & & & & & \\
\hline Agric. y ganad. & 112.704 & $44 \%$ & 109 & 6,3 & $52 \%$ & $1 \% \%^{* *}$ & $29 \%^{*}$ & $3 \% * *$ \\
\hline Industria & 152.157 & $61 \%$ & 687 & 9,2 & $42 \%$ & $13 \%^{*}$ & $17 \%^{*}$ & $7 \%^{*}$ \\
\hline Construcción & 146.903 & $38 \%$ & 312 & 7,3 & $47 \%$ & $9 \%^{*}$ & $20 \%^{*}$ & $10 \%^{*}$ \\
\hline Servicios & 140.968 & $62 \%$ & 1.462 & 10,3 & $41 \%$ & $8 \%^{*}$ & $17 \%^{*}$ & $10 \% *$
\end{tabular}

CLASE OCUPACIONAL

\begin{tabular}{lrrrrrrrr}
\hline Directivos & 298.970 & $50 \%$ & 46 & 13,1 & $30 \%$ & $14 \% * *$ & - & $3 \% * * *$ \\
\hline Técnicos & 179.882 & $61 \%$ & 551 & 13,2 & $28 \%$ & $\mathbf{6 \% *}$ & - & $9 \% *$ \\
\hline Empleados & 123.848 & $52 \%$ & 698 & 9,9 & $37 \%$ & $2 \% *$ & - & $16 \% *$ \\
\hline Manual calif. & 143.639 & $47 \%$ & 985 & 7,5 & $43 \%$ & $2 \% *$ & - & $8 \% *$ \\
\hline Manual sin calif. & 103.021 & $51 \%$ & 384 & 7,4 & $45 \%$ & $5 \% *$ & - & $2 \% * *$
\end{tabular}

TAMAÑO DE LA EMPRESA

\begin{tabular}{lrrrrrrrl}
\hline Pequeño & 117.142 & $46 \%$ & 777 & 8,7 & $45 \%$ & $5 \% *$ & $15 \% *$ & $4 \% *$ \\
\hline Mediano & 141.405 & $48 \%$ & 1.033 & 9,2 & $45 \%$ & $7 \% *$ & $22 \% *$ & $8 \% *$ \\
\hline Grande & 179.315 & $65 \%$ & 641 & 10,6 & $40 \%$ & $10 \% *$ & $18 \% *$ & $7 \% *$ \\
\hline \multicolumn{2}{l}{ ANTIGÜEDAD EN LA EMPRESA } & & & & & & \\
\hline Reciente & 125.700 & $52 \%$ & 1.772 & 9,7 & $42 \%$ & $8 \% *$ & $16 \% *$ & - \\
\hline Media & 177.035 & $57 \%$ & 560 & 9,2 & $45 \%$ & $18 \% *$ & $13 \% *$ & - \\
\hline Antigua & 202.317 & $57 \%$ & 197 & 7,8 & $54 \%$ & $23 \% *$ & $24 \% *$ & - \\
\hline
\end{tabular}

\section{RELACIÓN LABORAL}

\begin{tabular}{|c|c|c|c|c|c|c|c|c|}
\hline Fijos & 161.166 & $54 \%$ & 1.563 & 9,7 & $44 \%$ & $11 \% *$ & $1 \% *$ & $5 \% *$ \\
\hline Discontinuos & 121.317 & $40 \%$ & 183 & 8,3 & $45 \%$ & $0 \%$ *** & $7 \% * *$ & $6 \% * *$ \\
\hline Eventuales & 110.146 & $48 \%$ & 769 & 8,9 & $45 \%$ & $1 \% * *$ & $6 \% *$ & $0 \%$ *** \\
\hline \multicolumn{9}{|c|}{ TAMAÑO DEL HÁBITAT } \\
\hline Pequeño & 132.201 & $45 \%$ & 561 & 8,2 & $46 \%$ & $2 \% * *$ & $20 \% *$ & $9 \% *$ \\
\hline Mediano & 141.183 & $63 \%$ & 1.371 & 9,3 & $45 \%$ & 9\%* & $13 \% *$ & $8 \% *$ \\
\hline Grande & 153.275 & $59 \%$ & 744 & 10,4 & $40 \%$ & $10 \% *$ & $22 \% *$ & $11 \% *$ \\
\hline
\end{tabular}

*Significación de la $F<0,001$.

** Significación de la $F<0,01$.

*** Significación de la $F=$ no significativa.

Fuente: Encuesta de Calidad de Vida en el Trabajo, de 1999.

Elaboración propia. 
- No tiene apenas trascendencia en las personas asalariadas que se ocupan de las labores domésticas (este dato no aparece en la tabla).

- La clase ocupacional de «empleados» y la de "obreros manuales» tampoco parece beneficiarse mucho del nivel de estudios.

Por el contrario, el nivel educativo llega a explicar los ingresos en una proporción alta en ciertas categorías:

— La rama de actividad industrial es la que más valora el nivel de estudios.

- Igual ocurre con la clase ocupacional de los «directivos». En el caso de los «técnicos», se aprecia menos la valoración debido a que casi todos tienen un alto nivel de estudios. Es decir, los estudios no son discriminantes en esta clase.

- La mayor antigüedad en la empresa es otra fuente de valoración positiva del nivel de estudios.

- Las empresas grandes y los hábitats grandes favorecen la rentabilidad, también, de la escolaridad.

\section{La importancia de la educación en el nivel de ingresos. Posibles explicaciones}

De acuerdo con los datos anteriores, la organización interna de la empresa justifica las diferencias de salarios mucho más que el nivel educativo de los empleados. Sin embargo, también es verdad que existe una gran variabilidad entre las categorías ocupacionales observadas. La proporción en que participa la educación en los citados ingresos difiere mucho de una categoría a otra.

La teoría de la modernización y su epígono, la teoría de la sociedad postindustrial (D. Bell, 1976), así como el funcionalismo tecnológico (B. Clark, 1962) y su correlato económico, la teoría del capital humano (G. Becker, 1983), se basan en la idea de que un nivel educativo alto se corresponde con una mayor productividad del empleado. Ahora bien, esta relación entre educación y productividad es más fuerte cuanto más alto sea, a su vez, el desarrollo económico y tecnológico del país, del sector de producción o de la empresa correspondiente. Se puede confundir la productividad, entonces, con un mayor nivel de desarrollo económico y tecnológico del puesto de trabajo.

En general es muy difícil medir el nivel de productividad de cualquier empleado individual, por eso se suele utilizar como variable "proxi» de tal productividad el nivel de ingresos, dando por supuesto que tal nivel de ingresos depende directamente de la capacidad productiva. Los datos que manejamos avalan la idea de que el nivel educativo tiene más importancia cuanto más alta sea la media de ingresos de la categoría observada. Considerando todas las categorías ocupacionales de la tabla 7 , se aprecia una alta correlación entre la media de ingresos y el coeficiente de determinación del nivel educativo sobre esos ingresos (coeficiente de correlación $=0,65$ ). Es decir que cuanto más alta sea la media de ingresos, mayor proporción de la variabilidad de los mismos es explicada por las diferencias educativas. 
Según la hipótesis inicial, por lo tanto, cuanta mayor productividad tenga la categoría ocupacional observada (o mayor nivel de desarrollo económico y tecnológico de la misma), mayor trascendencia tendrá el nivel educativo en la misma. Obsérvese, de paso, que la media de ingresos tiene muy poco que ver con el coeficiente de determinación del tipo de puesto en la empresa (dirección, supervisión y empleado) sobre tales ingresos (c. d. 2). El coeficiente de correlación correspondiente es muy bajo $(0,26)$. En otras palabras, la productividad (o el desarrollo económico y social) de cada categoría ocupacional no tiene que ver apenas con el criterio de salarización interno de la empresa.

La expansión educativa, por ahora, no es tampoco causa de deterioro de los ingresos de los más educados. La correlación entre la importancia económica del nivel educativo y la cantidad de años de escolaridad es positiva, aunque muy baja (c. c. $=0,24)$. Por su parte, la importancia económica de la organización interna de la empresa es totalmente independiente de la media de años de escolaridad de cada categoría (c. c. $=0,04)$. Es decir, la media de años de escolaridad de cada categoría social tiene poco que ver con la rentabilidad correspondiente de los niveles educativos.

\section{El ajuste con el puesto de trabajo}

M. I. García Espejo (1998: 16-23) recuerda que desde la perspectiva de la teoría del capital humano hay dos modelos teóricos que intentan justificar el posible desajuste entre nivel educativo y puesto de trabajo. El más antiguo es el modelo de «acoplamiento al puesto de trabajo» (B. Jovanovic, 1979). Existe tal desacoplamiento porque la información sobre el mercado no es perfecta y la búsqueda es costosa. La consecuencia de tal desajuste es una movilidad ocupacional más abundante hasta conseguir un mejor acoplamiento. El segundo tipo es más reciente: es el modelo de «movilidad profesional» (N. Sicherman y O. Galor, 1990). En este caso, la movilidad profesional sigue una trayectoria claramente acumulativa, de forma que cada etapa es un escalón formativo necesario para conseguir la siguiente. Según esto, los asalariados que siguen dichos trayectos, desarrollan una movilidad ocupacional más horizontal que vertical, se mueven dentro de una misma clase ocupacional (directivo, técnico, empleado, manual calificado y manual sin calificar).

Las anteriores explicaciones dan pie para comprender el funcionamiento del mercado laboral. Caben dos opciones extremas: el ajuste entre demanda y oferta de empleo es inmediato, o, en caso contrario, se producen una serie de etapas hasta que se llega a una situación aceptable. Por su parte, en el caso de estas supuestas etapas, es posible la existencia de otras dos realidades: los cambios se suceden dentro de una misma clase ocupacional o, por el contrario, las etapas se suceden pasando de una clase ocupacional a otra.

La mayoría de los asalariados del sector privado entrevistados han cambiado de empleo (65\%). Sin embargo, en esta categoría que cambia de empleo es más bajo el coeficiente de determinación de la educación sobre los ingresos (7\%), que en el caso de los que continúan en el mismo trabajo (10\%). Esto 
quiere decir que el ajuste inmediato con el puesto de trabajo es menos abundante, pero, al mismo tiempo, más rentable para el nivel educativo.

En la tabla 8 se proporciona la información pertinente según las categorías ocupacionales habituales. El coeficiente de correlación entre la columna primera (coeficiente de determinación de la educación sobre los ingresos) y la columna segunda (proporción de asalariados que ha cambiado de empleo) es negativo, si bien es muy bajo (-0,31). Esto significa que, dentro de unos límites reducidos, el cambio de empleo no favorece la rentabilidad de los estudios.

La movilidad intrageneracional entre clases ocupacionales sí parece influir en la rentabilidad de los estudios. Aproximadamente la mitad de los que cambian de empleo lo hacen dentro de la misma clase ocupacional. El coeficiente de determinación de la educación sobre los ingresos es inferior entre los que se mueven dentro de la misma clase ocupacional (5\%). Por el contrario, ese coeficiente asciende (10\%) cuando la movilidad se produce entre clases diferentes. Por otro lado, la correlación entre la columna primera de la tabla 8 (coeficiente de determinación del nivel educativo sobre los ingresos) y la columna tres (proporción de asalariados que se mueven dentro de la misma clase ocupacional) también es negativa, aunque ciertamente baja $(-0,32)$.

Los datos anteriores inducen a pensar que los modelos explicativos del desajuste desarrollados a partir de la teoría del capital humano no son correctos. En primer lugar, la rentabilidad de los estudios se consigue más abundantemente desde una primera contratación. Además, en caso de tener que cambiar de empleo, parece más rentable para el nivel educativo cambiar de clase ocupacional. En otras palabras, no hay formación acumulativa que sirva para los siguientes trabajos. La única formación acumulativa es la que se produce en el mismo trabajo.

\section{Epílogo}

Los modelos explicativos de la relación entre educación y empleo son muy variados. Además, la mayoría son de difícil verificación empírica. En este trabajo se propone un conjunto de clasificaciones dicotómicas de explicaciones de la citada relación. En cada conjunto dicotómico es fácil elegir el modelo que se corresponde mejor con la realidad.

La primera dicotomía es aquélla que se establece entre importancia del nivel educativo nacional versus importancia de la organización interna de la empresa. Los datos que manejamos de la Encuesta de Calidad de Vida en el Trabajo, de 1999, inducen a pensar que tiene mucha más importancia la organización interna de la empresa que el nivel educativo a la hora de determinar los ingresos de las asalariados del sector privado.

La segunda dicotomía es aquélla que distingue entre educación directamente productiva versus educación de interés productivo indirecto. En otras palabras, la productividad emana del nivel educativo exterior a la empresa o, por el contrario, del aprendizaje en el puesto de trabajo dentro de la empresa. La antigüedad en la empresa constituye un indicador básico en esta dicotomía. 
Tabla 8. Cambio de empleo y movilidad social intrageneracional.

Asalariados del sector privado

\begin{tabular}{lcccc}
\hline $\begin{array}{l}\text { Categoría } \\
\text { ocupacional }\end{array}$ & $\begin{array}{c}\text { c. d. 1 } \\
\text { (educac.) }\end{array}$ & $\begin{array}{c}\text { Han cambiado } \\
\text { de empleo }\end{array}$ & $\begin{array}{c}\text { Herencia } \\
\text { intragener. }\end{array}$ & $\begin{array}{c}\text { Herencia } \\
\text { intergener. }\end{array}$ \\
\hline Agricultura y ganadería & $1 \%$ & $54 \%$ & $58 \%$ & $66 \%$ \\
\hline Industria & $13 \%$ & $67 \%$ & $55 \%$ & $53 \%$ \\
\hline Construcción & $9 \%$ & $72 \%$ & $49 \%$ & $60 \%$ \\
\hline Servicios & $8 \%$ & $64 \%$ & $47 \%$ & $28 \%$ \\
\hline Directivos & $14 \%$ & $69 \%$ & $7 \%$ & $18 \%$ \\
\hline Técnicos & $6 \%$ & $61 \%$ & $46 \%$ & $27 \%$ \\
\hline Empleados & $2 \%$ & $64 \%$ & $52 \%$ & $18 \%$ \\
\hline Manual calificado & $2 \%$ & $67 \%$ & $59 \%$ & $70 \%$ \\
\hline Manual sin calificar & $5 \%$ & $69 \%$ & $32 \%$ & $25 \%$ \\
\hline Empresa pequeña & $5 \%$ & $63 \%$ & $49 \%$ & $38 \%$ \\
\hline Empresa mediana & $7 \%$ & $67 \%$ & $51 \%$ & $43 \%$ \\
\hline Empresa grande & $10 \%$ & $68 \%$ & $49 \%$ & $40 \%$ \\
\hline Antigüedad: reciente & $8 \%$ & $71 \%$ & $50 \%$ & $38 \%$ \\
\hline Antigüedad: media & $18 \%$ & $59 \%$ & $52 \%$ & $45 \%$ \\
\hline Antigüedad: antigua & $23 \%$ & $48 \%$ & $46 \%$ & $45 \%$ \\
\hline Fijos & $11 \%$ & $64 \%$ & $50 \%$ & $40 \%$ \\
\hline Discontinuos & $0 \%$ & $65 \%$ & $60 \%$ & $41 \%$ \\
\hline Eventuales & $1 \%$ & $69 \%$ & $48 \%$ & $44 \%$ \\
\hline Hábitat pequeño & $2 \%$ & $62 \%$ & $51 \%$ & $46 \%$ \\
\hline Hábitat mediano & $9 \%$ & $65 \%$ & $50 \%$ & $41 \%$ \\
\hline Hábitat grande & $10 \%$ & $68 \%$ & $47 \%$ & $36 \%$ \\
\hline E & $1 \%$ & & \\
\hline
\end{tabular}

Fuente: Encuesta de Calidad de Vida en el Trabajo, de 1999.

Elaboración propia.

Los datos indican que la antigüedad en la empresa es básica para rentabilizar el nivel de estudios. Es decir, que los estudios tienen interés empresarial indirecto, ya que la educación no es rentable desde un primer momento.

La tercera dicotomía es la que diferencia el modelo de producción indirecta favorable a la empresa versus el favorable al estatus del empleado. De acuerdo con la última parte de la disyuntiva, la educación es nada más que un mecanismo de mantenimiento de estatus familiar. La movilidad de clase ocupacional intergeneracional es una variable imprescindible en este análisis. El resultado del mismo indica que el modelo de estatus familiar del empleado 
tiene escaso interés. No explica la relación entre nivel educativo e ingresos apenas. Esta afirmación se sabía desde hacía tiempo.

De cualquier forma, hay categorías sociales y ocupacionales en las que el nivel educativo es muy rentable. Eso ocurre, por ejemplo, en la rama de producción industrial, en la clase ocupacional de "directivos», en los que tienen más antigüedad en la empresa, en las empresas de tamaño grande y en los hábitats más poblados.

En general, se produce una mayor rentabilidad de los estudios formales en las categorías ocupacionales que reciben los salarios más altos. Esto puede interpretarse indirectamente como que la educación es más rentable cuando el nivel de productividad es más alto, mayor es el desarrollo tecnológico y más elevado es el nivel de modernización.

El ajuste del nivel educativo y el empleo es complicado. Aquéllos que encajan a la primera obtienen una rentabilidad de sus estudios superior a la de los que lo intentan varias veces. Pero son una minoría. La mayoría lo intenta varias veces. Para éstos es más rentable para sus estudios cambiar de una clase ocupacional a otra, que cambiar dentro de la misma clase ocupacional. Es decir, no sirve de mucho la experiencia similar pero en empresas diferentes. Es más rentable, ya que se cambia de empresa, cambiar también de clase ocupacional. La experiencia en el puesto de trabajo es rentable para el nivel educativo si se produce en la misma empresa.

\section{Bibliografía}

Arrow, K. J. (1991). «La educación superior como filtro», Economía y Sociedad, 8, p. $143-161$.

BECKER, G. (1983). El capital humano, Madrid: Alianza.

Boudon, R. (1977). «Education and Social Mobility: A Structural Model», en

J. Karabel; A. H. Halsey (eds.), Power and Ideology in Education, Nueva York:

Oxford University Press, p. 186-196.

BOWLES, S.; GINTIS, H. (1985). La instrucción escolar en la América capitalista, Madrid: Akal.

Brown, H. Ph. (1990). Las desigualdades de salarios, Madrid: Ministerio de Trabajo y Seguridad Social.

BRUNeT ICART, I.; MORELl BlanCH, A. (1998). «Mercado de trabajo y estrategias de valorización", Revista Española de Investigaciones Sociológicas, 82, p. 37-71.

Carabaña, J. (1999). Dos estudios sobre movilidad intergeneracional, Madrid: Fundación Argentaria y Visor.

ClarC, B. (1962). Educating the Expert Society, San Francisco: Chandler.

Collins, R. (1989). La sociedad credencialista, Madrid: Akal.

Corugedo, I. (1995). «Productividad y empleo», Información Comercial Española, 743, p. 123-130.

Diéz Nicolás, J.; Pino ARTACHO, J. del (1972). «Estratificación y movilidad social en España en la década de los años 70", en CAMPO, S. del (dir.), La España de los años 70, vol. I, Madrid, p. 381-430.

Doeringer, P. B.; M. J. PIORE (1999). «Los mercados internos de trabajo», enTOHARIA, L. (comp.), El mercado de trabajo: Teorías y aplicaciones, Madrid: Alianza, p. 341-388. 
EDWARDS, R. C. (1999). «Las relaciones sociales de producción de la empresa y su estructura de mercado de trabajo", en TOHARIA, L. (comp.), El mercado de trabajo: Teorías y aplicaciones, Madrid: Alianza, p. 395-422.

Fina Sanglas, Ll.; Toharia Cortés, L.; García Serrano, C.; Mañé Vernet, F. (2000). "Cambio ocupacional y necesidades educativas de la economía española», en SÁEZ, F. (coord.), Formación y empleo, Madrid: Fundación Argentaria y Visor, p. 47-154.

GarCía EsPejo, M. I. (1998). Recursos formativos e inserción laboral de los jóvenes, Madrid: CIS.

Golsmith, A.H.; Veum, J.R.; Darity, J. Jr. (2000). «Psicología y productividad: estructuración de una relación efectiva entre empresario y trabajador», en OSUNA, R.; RomÁn, C., El problema del empleo en el siglo XXI, Málaga: Instituto de Desarrollo Regional, p. 133-149.

Jovanovic, B. (1979). "Job Matching and the Theory of Turnover», Journal of Political Economy, 87, 5, p. 973-989.

KaTZ, M. L.; Rosen, H. L. (1997). Microeconomía, Madrid: McGraw Hill.

MARSDEn, D. (1994). Mercados de trabajo. Limites sociales de las nuevas teorías, Madrid: Ministerio de Trabajo y Seguridad Social.

PeINAdo López, A. (1988). La discriminación de la mujer en el puesto de trabajo español: Una aproximación empirica a la discriminación salarial, Madrid: Ministerio de Trabajo y Seguridad Social.

Piore, M. J. (1999). «Notas para una teoría de la estratificación del mercado de trabajo», en TOHARIA, L. (comp.), El mercado de trabajo: Teorías y aplicaciones, Madrid: Alianza, p. 193-222.

Rubinson, R.; Browne, I. (1994). "Education and Economy», en SMELSER, N. J.; Swedberg, R. (eds), The Handbook of Economic Sociology, Princenton y Nueva York: Princenton University Press y Russell Sage Foundation, p. 581-599.

Ruesga Benito, S. M.; García de la Cruz, J. M.; Muruyama Rendón, C. (2000). «Introducción: La relación entre educación y empleo. Una aproximación a su análisis económico», en SÁEZ, F. (coord.), Formación y empleo, Madrid: Fundación Argentaria y Visor, p. 19-46.

SiCHERMAN, N.; GALOR, O. (1990). "A theory of career Mobility», Journal of Political Economy, 98, 1, p. 160-192.

Thurow, L. C. (1977). «Education and Economic Equality», en J. Karabel; A. H. HalSEY (eds.), Power and Ideology in Education, Nueva York: Oxford University Press, p. 325-334.

TRIPIER, P. (1995). Del trabajo al empleo. Ensayo de sociología de la sociología del trabajo. Madrid: Ministerio de Trabajo y Seguridad Social. 